\title{
Aborto entre alunas e funcionárias de uma universidade brasileira*
}

\section{Abortion among female students and employees of a brazilian university}

\author{
Ellen Hardy**, Ivanise Rebello**, Aníbal Faúndes**
}

\begin{abstract}
HARDY, E. et al. Aborto entre alunas e funcionárias de uma universidade brasileira. Rev. Saúde Pública, 27: 113-6, 1993. No Brasil existem poucas informaçōes sobre o aborto provocado e suas características na população. A dificuldade em se obter dados confiáveis deve-se: à sua ilegalidade, e também ao fato de que a maioria dos estudos é realizada em hospitais. Assim, em 1990, foi realizada pesquisa entre alunas da graduação e funcionárias de uma universidade brasileira. Os dados foram obtidos por meio de questionário, auto-respondido, para devolução por correio, anonimamente. Os resultados apresentados referem-se à frequêencia do aborto provocado nesta população. Encontrou-se que quatro vezes menos alunas do que funcionárias tinham engravidado alguma vez $(15 \%$ e $65 \%) ; 9 \%$ das alunas e $14 \%$ das funcionárias tiveram aborto provocado. Entre as mulheres que engravidaram alguma vez, as alunas tiveram mais aborto provocado do que as funcionárias (59\% e $20 \%$ ). Quando controladas por grupo etário as diferenças entre as alunas e funcionárias mantiveram-se. Entretanto, entre as alunas, o aborto provocado foi mais frequiente entre as mais jovens.
\end{abstract}

Descritores: Aborto induzido, estatística. Fatores etários.

\section{Introduçáo}

No Brasil o assunto do aborto provocado é muito controvertido e considerado por alguns como um dos principais responsáveis por um número importante de mortes maternas por infecção $\left(\right.$ Neme $\left.^{6}, 1991\right)$. Entretanto, existem poucas informações sobre a frequiência com que ocorrem os abortos provocados e as características das mulheres que abortam. Isso se deve ao fato de que a maioria das pesquisas tem sido realizada em hospitais-escolas ou maternidades que atendem prioritariamente a população de baixa renda e onde só chegam os casos com complicaçōes (Fariña ${ }^{3}$, 1975; Boehs e col. ${ }^{1}, 1984$; Schor $\left.{ }^{9}, 1987\right)$. As estimativas de abortos provocados por ano variam de 4 milhőes a 500 mil. Os dados do Instituto $\mathrm{Na}$ -

\footnotetext{
* Pesquisa parcialmente financiada pela Universidade Estadual de Campinas (UNICAMP), Programa Latinoamericano de Capacitación e Investigación en Reprodución Humana (PLACIRH), International Women's Health Coalition (IWHC) e The Population Council.

** Departamento de Tocoginecologia, Faculdade de Ciências Médicas, Universidade Estadual de Campinas (UNICAMP) - Centro de Pesquisa e Controle das Doenças Materno-Infantis de Campinas (CEMICAMP) - Cidade Universitária Zeferino Vaz - Campinas, SP . Brasil.
}

Separatas/Reprints: E. Hardy - Caixa Postal 6181 - 13081-970 Campinas, SP - Brasil.

Edição subvencionada pela FAPESP. Processo Medicina 93/ 0208-5. cional de Assistência Médica e Previdência Social (INAMPS) para 1980 mostraram que mais de 200 mil mulheres foram hospitalizadas por complicações de abortos. Considerando-se que nem todos os abortos provocados resultam em hospitalização e que nem sempre os arquivos hospitalares registram o problema, pode-se imaginar que os dados oficiais apresentam-se defasados em relação à realidade (Pinotti e Faúndes ${ }^{8}, 1989$ ).

Estudo realizado com 234 pacientes que deram entrada em um hospital com diagnóstico de abortamento constatou que $34 \%$ delas admitiu ter provocado o aborto. Dentre essas mulheres, $29 \%$ referiu já ter feito um aborto antes (Pinotti e col. ${ }^{7}, 1969$ ).

Galbinski e col. ${ }^{4}$ (1971) constataram que de 5.183 gestações, 1.428 foram abortos, dos quais 776 provocados, bem como estudaram mais detidamente 1.211 mulheres de 15 a 49 anos de idade e com vida sexual ativa, observando que $25,1 \%$ tinham antecedentes de aborto provocado.

Pesquisa realizada em uma maternidade constatou 1.615 admissões, sendo que 351 eram abortamentos, dos quais $22,5 \%$ provocados. Das mulheres que tinham provocado o aborto, a maioria estava na faixa de 20 a 24 anos de idade (Farias 2, 1972).

Em uma pesquisa hospitalar onde se coletaram informações dos prontuários de 2.588 pacientes, encontrou-se que $o$ atendimento a mulheres com complicaçס̃es do aborto sofre progressivo aumento, duplicando o seu número entre 1978 e 1982, o que não se verificou em relação ao número de partos que teve um aumento de apenas $14 \%$ (Schor $\left.{ }^{9}, 1984\right)$. 
Um dos obstáculos mais importantes para a obtenção de dados confiáveis sobre aborto provocado é sua ilegalidade, o que dificulta pesquisá-lo através de entrevistas. As mulheres podem não ficar a vontade para admitir um aborto e relatar sua experiência quando se veêm pela primeira vez, cara a cara com uma entrevistadora. Um procedimento que permitiria que a mulher ficasse mais confortável seria realizar a pesquisa através de questionário que ela mesma preencheria com a garantia de que não seria identificada. Os resultados desse tipo de pesquisa, entretanto, dependem muito da motivação da pessoa que recebe o questionário: para lê-lo, preenchê-lo e devolvê-lo ao pesquisador, pelo correio.

Diante disto, foi escolhida uma população universitária para desenvolvimento do estudo, pois acredita-se que haveria mais motivação para responder o questionário, considerando-se que teriam maior familiaridade com pesquisa do que a população em geral. A finalidade do presente estudo $E$ analisar a freqüência de aborto em diferentes idades, comparando alunas e funcionárias.

\section{Material e Método}

A presente pesquisa foi desenvolvida em uma universidade do Estado de São Paulo, em 1990, sendo a população constituída de todas as funcionárias e alunas de cursos de graduação. Os dados foram obtidos por meio de questionário autorespondido, pré-testado em uma das faculdades da universidade. Junto com o questionário foram distribuídos: uma carta explicando a pesquisa e um envelope carta-resposta comercial. Maiores detalhes sobre a metodologia já foram anteriormente publicadas (Hardy e col.5, 1991).

Foram recebidos 1.992 questionários respondidos pelas funcionárias e 937 pelas alunas, o que correspondeu a $27 \%$ e $42 \%$ do total distribuido, respectivamente.

\section{Resultados}

Quase $10 \%$ das alunas e uma quarta parte das funcionárias responderam que alguma vez tiveram um aborto. Aproximadamente $9 \%$ das alunas e $13 \%$ das funcionárias declararam ter tido pelo menos um aborto provocado. Menos alunas do que funcionárias haviam tido mais de um aborto provocado. Todas essas diferenças foram significativas (Tabela 1).

A percentagem de alunas e de funcionárias com antecedentes de aborto foi significativamente menor entre as mulheres com até 24 anos de idade
Tabela 1. Histórico de abortos (em percentagem) **

\begin{tabular}{lccc}
\hline Histórico de aborto & Alunas & Funciónarias & Total \\
\hline Aborto * & 9,7 & 25,4 & 20,4 \\
Aborto Provocado \# & 8,8 & 13,5 & 12,0 \\
N2 de Aborto & & & \\
Provocado + & & & \\
1 & 7,2 & 8,5 & 8,0 \\
2 & 1,1 & 2,6 & 2,1 \\
3 & 0,3 & 1,2 & 0,9 \\
4 ou mais & 0,2 & 0,8 & 0,6 \\
(N) & 937 & 1.992 & 2.929 \\
\hline
\end{tabular}

- $p<0,00001 ; \# p<0,0004 ;+<0,0008$

* Percentagens calculadas excluindo as mulheres sem informaçảo.

comparadas com as de 25 anos ou mais. Dentro de cada grupo de idade já não se observaram diferenças entre alunas e funcionárias (Tabela 2). Ao comparar o antecedente de aborto provocado, as mesmas diferenças por idade foram observadas tanto entre alunas quanto entre funcionárias. Não houve diferença significativa entre a percentagem de alunas e funcionárias com aborto provocado dentro de cada grupo de idade (Tabela 3 ).

Tabela 2. Percentagem de alunas e funcionárias com antecedentes de aborto, segundo idade por ocasiāo do estudo

\begin{tabular}{lccccc}
\hline Idade & \multicolumn{2}{c}{ Alunas } & \multicolumn{4}{c}{ Funcionárias } & $p$ \\
\hline & $\%$ & $n$ & $\%$ & $n$ & \\
Até 24 anos & $7,1^{*}$ & $(807)$ & $7,9^{*}$ & $(253)$ & N.S. \\
25 anos ou mais & 27,4 & $(124)$ & 28,4 & $(1.601)$ & N.S. \\
\hline
\end{tabular}

"Até 24 vs 25 anos ou mais: $p<0,0001$

Tabela 3. Percentagem de alunas e funcionárias com antecedentes de aborto provocado segundo, idade por ocasião doestudo.

\begin{tabular}{lccccc}
\hline Idade & \multicolumn{2}{c}{ Alunas } & \multicolumn{2}{c}{ Funcionárias } & $p$ \\
\hline & $\%$ & $n$ & $\%$ & $n$ & \\
& & & & & \\
Até 24 anos & $6,9^{*}$ & $(807)$ & $4,3^{*}$ & $(253)$ & N.S. \\
25 anos ou mais & 21,0 & $(124)$ & 15,4 & $(1.602)$ & N.S. \\
\hline
\end{tabular}

- Até 24 vs 25 anos ou mais: $p<0,0001$

Ao estudar 0 aborto somente entre as mulheres que alguma vez estiveram grávidas observou-se que significativamente mais alunas $(65,9 \%)$ do que funcionárias $(38,2)$ haviam tido pelo menos um aborto. A diferença aumentou ao analisar as que tiveram pelo menos um aborto provocado, sendo que as alunas apresentaram percentagem 
quase três vezes superior às funcionárias. As diferenças foram significativas (Tabela 4).

Tabela 4. Antecedentes de aborto e aborto provocado entre alunas $\theta$ funcionárias que alguma vez estiveram grávidas.

\begin{tabular}{|c|c|c|c|c|c|}
\hline \multirow[t]{2}{*}{ Antecedentes } & \multicolumn{2}{|c|}{ Alunas } & \multicolumn{2}{|c|}{ Funcionárias } & \multirow[t]{2}{*}{$p$} \\
\hline & $\%$ & n & $\%$ & $n$ & \\
\hline $\begin{array}{l}\text { Aborto } \\
\text { Aborto Provocado }\end{array}$ & $\begin{array}{l}65,9 \\
59,4\end{array}$ & $\begin{array}{l}(138) \\
(138)\end{array}$ & $\begin{array}{l}38,2 \\
20,3\end{array}$ & $\begin{array}{l}(1.314) \\
(1.314)\end{array}$ & $\begin{array}{l}<0,00001 \\
<0,00001\end{array}$ \\
\hline
\end{tabular}

As diferenças entre alunas e funcionárias mantiveram-se quando separadas por grupos etários. No grupo de até 24 anos, o dobro de alunas (74\%) em relação às funcionárias $(35,7 \%)$ tiveram antecedente de aborto. No grupo de maior idade também houve mais aborto entre as alunas do que entre as funcionárias $(56,7 \%$ e $38,8 \%$ respectivamente). Não houve diferença significativa ao comparar os dois grupos de idade, tanto entre as alunas como entre as funcionárias (Tabela 5).

Tabela 5. Percentagem de alunas e funcionárias grávidas alguma vez, com antecedentes de aborto, segundo idade por ocasião do estudo.

\begin{tabular}{lccccc}
\hline Idade & \multicolumn{2}{c}{ Alunas } & \multicolumn{4}{c}{ Funcionárias } & $p$ \\
\hline & $\%$ & $n$ & $\%$ & $n$ & \\
& & & & & \\
Até 24 anos & $74,0^{*}$ & $(77)$ & 35,7 & $(56)$ & 0,00001 \\
25 anos ou mais & 56,7 & $(60)$ & 38,8 & $(1.169)$ & 0,00591 \\
\hline
\end{tabular}

- Até 24 vs 25 anos ou mais: NS

Ao estudar o antecedente de aborto provocado observaram-se as mesmas diferenças significativas ao comparar alunas e funcionárias dentro de cąda grupo etário. Entretanto, entre as alunas, também houve diferença ao comparar os dois grupos etários: 0 antecedente de aborto provocado foi significativamente mais frequiente no grupo de até 24 anos de idade (Tabela 6).

Tabela 6. Percentagem de alunas e funcionárias grávidas alguma vez, com antecedentes de aborto provocado, segundo idade por ocasiāo do estudo.

\begin{tabular}{lccccc}
\hline Idade & \multicolumn{2}{c}{ Alunas } & \multicolumn{4}{c}{ Funcionárias } & $p$ \\
\hline & $\%$ & $n$ & $\%$ & $n$ & \\
Até 24 anos & $72,7^{*}$ & $(77)$ & 19,6 & $(56)$ & 0,00000 \\
25 anos ou mais & 43,3 & $(60)$ & 21,0 & $(1.170)$ & 0,00005 \\
\hline
\end{tabular}

- Até 24 vs 25 anos ou mais: $p<0,00001$

\section{Discussáo}

Os resultados encontrados revelaram que uma percentagem relativamente alta das mulheres do grupo estudado tinha vivido a experiência de um aborto provocado. Essa percentagem foi ainda maior ao considerar apenas as mulheres alguma vez grávidas, entre as quais mais da metade das alunas, e uma de cada cinco funcionárias tinham tido pelo menos um aborto provocado. A importância desses dados reside na falta de informaçðes confiáveis sobre $o$ assunto, já que a maioria, dos poucos estudos referidos na literatura, apresenta resultados de pesquisas feitas em hospitais.

A metodologia utilizada para este estudo deve ter influenciado os resultados e a garantia do sigilo pode ter favorecido a sinceridade das respostas. Por outro lado, permitir às mulheres preencher suas respostas e enviar o questionário pelo correio significsu uma percentagem de não-resposta maior do que teria ocorrido, provavelmente, se fosse realizado um inquérito. Não é possivel saber se as m.ulheres que não responderam tiveram mais ou menos abortos provocados do que as mulheres que responderam. E provável que as mulheres com antecelente de aborto provocado, que não tenham entend:do corretamente a garantia do sigilo, se sentissem constrangidas ou com vergonha de responder. A não compreensão do sigilo é uma hipótese possivel, já que entre as funcionárias foi maior a percentagem de respostas de mulheres com pelo menos alguma educação universitária.

É possivel também pensar que as mulheres que não tinham a experiência do aborto provocado responderam menos porque não entenderam que se esperava da resposta de todas as alunas e funcionárias, ainda que não tivessem abortado. Por isso mesmo poderiam não estar interessadas no assunto. Nesse caso haveria mais mulheres sem aborto entre as que não responderam. Por outra parte, o predomínio de respostas de mulheres com alguma educação universitária faz com que os resultados não sejam representativos das mulheres em geral e sim de uma população com maior nível de escolaridade.

À primeira vista parecia que as funcionárias apresentaram maior proporção de abortos e abortos provocados que as alunas, entretanto, ao se fazer o controle por grupo etário, verificou-se que esta diferença era apenas resultadọ da maior idade do primeiro grupo. Não surpreende que as mulheres de maior idade tinham relatado, tanto mais abortos em geral, quanto abortos provocados, já que tiveram mais tempo de vida para passarem por estas experiências.

Porém, ao analisar a experiência de aborto e aborto provocado apenas entre aquelas mulheres que tiveram pelo menos uma gravidez, o quadro mudou. As alunas apareceram com maior pro- 
porção de abortos e abortos provocados que as funcionárias, e estas diferenças mantiveram-se quando controladas por grupo etário. Por outro lado, quanto menor a idade das alunas que responderam, maior a percentagem com aborto provocado entre aquelas alguma vez grávidas. Isto significa que, quando ocorreu a gravidez, as alunas tiveram maior tendência a abortar, e quanto menor a idade em que essa gestação ocorreu, maior a probabilidade que resultasse em aborto provocado. De fato, apenas uma aluna menor de 25 anos teve aborto não-provocado, entre aquelas alguma vez grávidas.

Aparentemente a gravidez não planejada é vista como um problema maior pelas mulheres que ainda estão estudando, do que por aquelas que já estão estabilizadas profissionalmente. Considerando-se que não sabemos quando aconteceram os abortos relatados pelas funcionárias, e que alguns poderiam ter ocorrido quando ainda eram estudantes, a diferença descrita poderia ser ainda maior, mas não menor que a observada. É também possível que as alunas tenham melhores condiçōes econômicas e familiares que uma parte das funcionárias, $\mathrm{e}$ isto tenha facilitado a resolução do problema através da indução do aborto.

De qualquer forma, o fato de que $59 \%$ das alunas alguma vez grávidas e $73 \%$ entre as menores de 25 anos, tenham tido pelo menos um aborto provocado, mostra a absoluta necessidade de prevenir a ocorrência da gravidez indesejada, e evidencia que o aborto provocado é uma realidade cotidiana, pelo menos nesse grupo social.

A ênfase nas alunas não deve ofuscar o problema das func:onárias, visto que uma de cada cinco das que estiveram alguma vez grávidas já provocou um aborto, independentemente da idade. Isto significa que, também entre mulheres estabilizadas profissionalmente, a gravidez indesejada, a ponto de levar ao aborto, é um fenômeno frequisente que deve ser evitado.

Acreditamos que esses dados, com todas as suas limitações, constituem-se em mais uma evidência, bastante sólida, de que o aborto provocado é uma realidade que atinge com frequêência alarmante a mulher brasileira, e que, portanto, merece um tratamento mais sério e aberto, do que aquele que tem recebido até agora em nossa sociedade.

\section{Agradecimentos}

Ao estatístico Sergio Vera e ao analista de sistema Carlos Mora.

HARDY, E. et al. [Abortion among female students and employees of brazilian university]. Rev. Saúde Pública,
27: 113-6, 1993. In Brazil, the subject of induced abortion is controversial and considered by some to be a serious public health problem. On the other hand there are little data available as to its frequency and general characteristics. The difficulty encountered in obtaining reliable information is to be explained by the illegality of abortion that inhibits women from talking about their experience, and most studies are carried out in hospitals and thus succeed in identifying only those women who have complications. A study was carried out in 1990 , involving all the female graduate students and employees of a Brazilian university. Data was obtained through a questionnaire that was returned by mail, anonymously. This paper presents some of the results relating to the frequency of miscarriage and abortion in this population. Significantly more students than employees were less than 25 years old ( $85 \%$ and $13.7 \%$ respectively); fewer students were married or in a common-law union (11\% of students as against $56 \%$ of employees) and four times fewer students than employees had never been pregnant ( $15 \%$ and $65 \%)$. Nine percent of the students and $14 \%$ of the employees had had at least one abortion. When only sometime pregnant women were taken into consideration, over half the students $(59 \%)$ and $20 \%$ of the employees had had an abortion. Differences between the two groups were maintened when considered by age, both for miscarriage and abortion. Students of less than 25 years of age presented the highest percentage of abortion.

Keywords: Abortion. Age factors.

\section{Referênclas Bibliográficas}

1. BOEHS, A. E. et al. Aborto provocado: estudo epidemiologico descritivo numa matemidade de Florianópolis, Santa Catarina. Ciênc. e Cul., 3: 502-6, 1983.

2. FARIAS, F. C. Condicionamentos socioeconômicos do abortamento provocado. Rev. Paul. Hosp., 20: 25-31, 1972.

3. FARINA, E. B. Estudos dos motivos, processos e consequiências do abortamento em população assistida no Pronto-Socorro Obstétrico do Amparo Matemal. Rev. Esc. Enferm. USP, 9: 323-46, 1975.

4. GALBINSKI, J. A. et al. Contribuiçãoao estudo da epidemiologia do aborto provocado. J.Bras.Ginec ., 71:201-8,1971.

5. HARDY, E. et al. Aborto provocado: diferenças entre pensamento e ação. Rev. Ginec. Obstet., 2: 111-6, 1991.

6. NEME, E. \& NEME, B. Mortalidade matema: causas e fundamentos para sua prevenção. In: Faúndes, A. \& Cecatti, J. G., eds. Morte materna: uma tragédia evitável. Campinas, Editora da UNICAMP, 1991. p. 199-217.

7. PINOTTI, J. A. et al. Contribuiçôes ao estudo médico-social das causas determinantes do abortamento provocado. Matern. e Inf., 28: 9-20, 1969.

8. PINOTTI, J. A. \& FAÚNDES, A. Unwanted pregnancy: challenges for health policy. Int. J. Gynecol. Obstet., (Suppl 3): 97-102, 1989.

9. SCHOR, N. Aborto como questão de saúde pública: estudo da demanda de mulheres que recorreram ao hospital por complicações do aborto. São Paulo, 1984. [Tese de Doutorado - Faculdade de Saúde Pública da USP].

Recebido para publicação em 4.5 .1992 Reapresentado em 10.11.1992 Aprovado para publicação em 8.2.1993 